\title{
IRIS RECOGNITION ALGORITHM BASED ON COMPLEX GEOMETRICAL SHAPE COVER
}

\author{
Hao Feng ', Wenming $\mathrm{Cao}^{2,3}$, Shoujue Wang ${ }^{3}$ \\ Jiaxing College Jiaxing, zhejiang, 314001,China, ${ }^{2}$ The College of Information \\ Engineering, ZheJiang University of Technology, Hangzhou, 310014, China, ${ }^{3} \mathrm{Lab}$ of \\ Artificial Neural Networks, Institute of Semiconductors, CAS, Beijing, 100083, \\ Chinacsann@zjut.edu.cn
}

Abstract: In this my paper, we present a kind of Iris Recognition based on Complex Geometrical Shape Cover. From the cognition science point of view, we constructed a Complex Geometrical Shape for iris recognition. In this method, irises are trained as "cognition" one class by one class, and it doesn't influence the original recognition knowledge for samples of the new added class. The results of experiments show the correct rejection rate is $98.9 \%$, the correct cognition rate and the error recognition rate are $95.71 \%$ and $3.5 \%$ respectively. The experimental results demonstrate that the correct rejection rate of the test samples excluded in the classes of training samples is very high. It proves the proposed method for iris recognition is effective.

Key words: Iris recognition, Complex Geometrical Shape Cover, HDS.

\section{INTRODUCTION}

With the appearance of information science field such as computer media signal processing etc., researchers get to look for various methods for processing multidimensional signal, the multidimensional signal processing theory therefore arises. However modern signal processing techniques, mostly depending on the complicated mathematical formulas, look for an optimum object by means of iteration and fitting etc. These methods have 
some disadvantages including huge computation load, non direct-viewing, hard to be understood, moreover often trapping at local minima.

In the recent years, Wang Soujue[1-4] proposed complex geometrical shape cover method, which uses the high dimensional space descriptive geometry as tools, maps multidimensional digital signals to be points in HDS, and discuss relationship among multidimensional digital signals via analysis of point distribution and geometric object characteristics in HDS. Since this method is simple and feasible, and is able to accelerate computation by parallel operation, it has get remarkable achievements in many application fields [4,5-14].

In the light of descriptive geometry and notions in set theory, this paper redefines the basic elements in space such as curve and surface and so on, presents some fundamental notions with respect to the point cover based on the HDS point covering theory, finally, as an example, considers points generated by mapping part of speech signals to HDS to analyze distribution information of these iris sample in HDS, in this paper also proposes a new algorithm for iris recognition on the basis of the HDS point dynamic searching theory.

The remainder of this paper is organized as follows. Section 2 describes the theory of multi weights neurons. Section 3 introduces iris recognition algorithm based on Complex Geometrical Shape Cover and multi-weighted neuron. Experiments results and experimental analysis is effective.

\section{THE THEORY OF MULTI WEIGHTS NEURONS}

As we said in the former, the method used by BPR is "Complex Geometrical Shape Cover Recognition Method". The present neurons are based on single-weight vector which can only reach the simple and regular shapes. In fact, the real shapes of some a pattern in the feature space, such as a given person's face images, are irregular and complicated, so we developed a novel theory of multi-weight vector neurons which can reach the complicated shapes and meet the need of BPR.

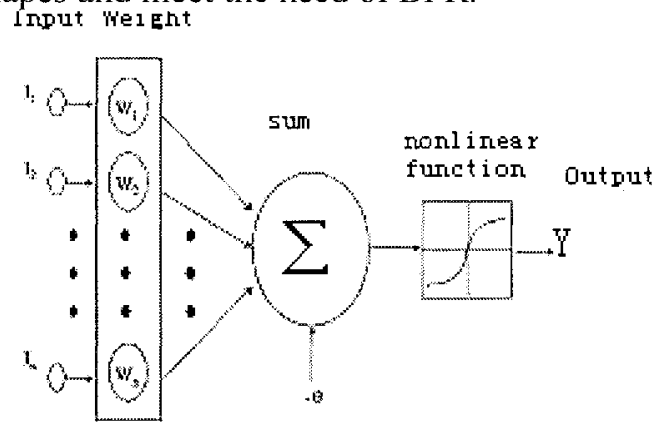




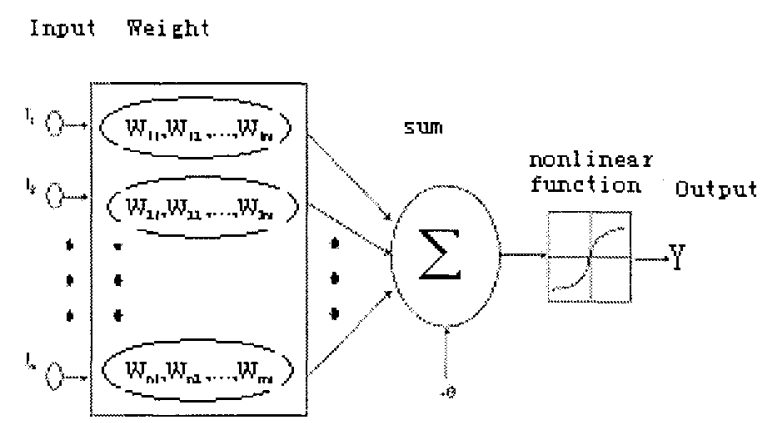

Fig 2.Multi Weight Neuron Model

According to the theory of BPR, the different samples of the same class are connected, then we can know all of the same class samples should be in a irregular and connected region. The aim of pattern recognition is to find this region or use a geometry shape to approximate to it as possibly. DBF is one typical method which use "hypersphere" to approximate real shape[6][7][18], however, few real shapes decided by the same class samples exactly are hypersphere. In our theory, all samples of the same class should be included in the shape covered by neuron, so when this shape is different from the real shape, it must be bigger than the real one and it not only covers samples of this class but also covers other class samples which should not be covered, then the error recognition rate will increase. Differently, the different approximation will result in different error recognition rate.

Generally, the expression of a multi-weight vectors neuron is:

$$
Y=f\left[\Phi\left(W_{1}, W_{2}, \ldots, W_{m}, X\right)\right]
$$

$W_{1}, W_{2}, \ldots, W_{m}$ are $\mathrm{m}$ weights vectors and are decided by a group of study samples vectors $S_{1}, S_{2}, \ldots, S_{m} ; X$ is the input vector; $\Phi$ is a function decided by a multi weight neuron; $f$ is step function, which is equal to 1 when $\mathrm{x}<=\mathrm{k}$ and equal to 0 when $\mathrm{x}>\mathrm{k}$.

\section{THE APPLICATION FOR IRIS RECOGNITION OF MULTI WEIGHTS NEURONS}

In recent years, with the development of information technology and the increasing need for security, intelligent personal identification has become a very important and urgent problem. The emerging biometric 
technology can solve the problem, which takes the unique, reliable and stable biometric features (such as fingerprints, iris, face, palm-prints, gait etc.) as identification body. This technology has very high security, reliability and effectivity. As one of the biometric technology, iris recognition has very high reliability. Comparing with other biometric identification technology, the fault acceptance rate and the fault rejection rate of iris recognition are very low. The technology of iris recognition has many advantages, i.e., stability, non-invasiveness, uniqueness. All there desirable properties make the technology of iris recognition has very high commercial value. Based on the above reasons, many researchers have applied themselves to this field. Daugman used multi-scale quadrature wavelets to extract texture-phase structure information of iris to generate a 2048-bit iriscode and compared the difference between a pair of iris representations by computing their Hamming distance via the XOR operator [8],[9]. Wildes et al. represented the iris texture with a Laplacian pyramid constructed with four different resolution levels and used the normalized correlation to determine whether the input image and the model image are from the same class [10]. Boles et al. calculated zero-crossing representation of $1 \mathrm{D}$ wavelet transform at various resolution levels of a virtual circle on an iris image to characterize the texture of the iris. Iris mating was based on two dissimilarity functions [11]. As follow, Section3.1 describes Image preprocessing of iris-- Iris localization, Localization of the inner boundary, Localization of the outer boundary, Iris normalization and enhancement. Section 3.2 introduces iris recognition algorithm based on multi weight neuron, Experiments results and experimental analysis.

\subsection{Image preprocessing}

Iris image preprocessing is mainly composed of iris localization, iris normalization and enhancement.

Iris localization namely is the localization of the inner boundary and the outer boundary of a typical iris can approximately be taken as circles. It is the important part of the system of iris recognition, and exact localization is the premise of the iris identification and verification.

The original iris image has some character of the gray-scale distribution. The iris is darker than the sclera, and the pupil is greatly darker than the iris. From the histogram, we can clearly see that the low gray-scale mainly converges at the first peak value. Therefore, we adopt the binary transform to localize the inner boundary. From the image after binary transform, we find that the areas of zero gray-scale are almost the areas of the pupil and eyelash. Therefore, we reduce the influence of the eyelash by erode and dilation. 


$$
x \text { pupil }=x \text { begin }+\frac{\text { dia } \max }{2}, y \text { pupil }=y \text { begin }, \text { rpupil }=\frac{\text { dia } \max }{2} 。
$$

Where xpupil and ypupil denote the center coordinates of the pupil, and rpupil denotes the radius of the pupil. after erode and dilation

When the quality of the image is reliable, this algorithm can localize the pupil quickly and exactly. Otherwise, we can correct the method as follow:

1. We can reduce the searching area by subtracting the pixels on the edge of the image.

2. We can get $k$ chords, which are less than a certain threshold near the longest chord, and take the average value of center coordinates of $k$ chord as the center of the pupil.

\subsection{Localization of the outer boundary}

The exact parameters of the outer boundary are obtained by using edge detection (Canny operator in our experiments) and Hough transform. The image after Edge detection includes some useless points. For eliminating the influence, we remove the useless points between the areas of $\left[30^{\circ}, 150^{\circ}\right]$ and $225^{\circ}, 315^{\circ}$ according to the center of the pupil. Then, Hough transform [14] is adopted to localize the outer boundary.

\subsection{Iris normalization and enhancement}

Irises from different people may be captured in different size, and even for irises from the same eye, the size may change because of illumination variations and other factors (the pupil is very sensitive to lighting changes). Such elastic deformation in iris texture will influence the results of iris recognition. For the purpose of achieving more accurate recognition results, it is necessary to compensate for such deformation. In our experiment, every point of the iris image is mapped to the polar coordinates by the following formula.

$$
\left\{\begin{array}{l}
x(r, \theta)=(1-r) x_{p}(\theta)+r x_{s}(\theta) \\
y(r, \theta)=(1-r) y_{p}(\theta)+r y_{s}(\theta)
\end{array}\right.
$$

In which, $\left(x_{p}(\theta), y_{p}(\theta)\right)$ and $\left(x_{s}(\theta) y_{p}(\theta)\right)$ denote the point of intersection with the inner boundary and the outer boundary respectively.

In our experiment, the sector areas $\left(\left[130^{\circ}, 230^{\circ}\right]\right.$ and $\left.\left[310^{\circ}, 410^{\circ}\right]\right)$ are intercepted for normalization according the pupil center. In this way, one hand, it is simple; on the other hand, the segment texture information is enough to identify the different persons. Then, the iris ring is unwrapped to a rectangular texture block with a fixed size $(64 \times 256)$, and the rows 
correspond to the radius and the columns correspond to the angles. The normalized iris image still has low contrast and may have non-uniform brightness caused by the position of light sources. All these may affect the feature analysis. Therefore, we enhance the normalized image by means of histogram equalization. Such processing compensates for non-uniform illumination, as well as improving the contrast of the image.

\subsection{Iris recognition algorithm based on Complex Geometrical Shape}

Complex Geometrical Shape, $p S i 3$ neuron, can be described as follow:

$$
\begin{aligned}
& Y=f\left[\Phi\left(X, W_{1}, W_{2}, W_{3}\right)-T h\right] \\
& \Phi\left(X, W_{1}, W_{2}, W_{3}\right)=\left\|X-\theta_{\left(W_{1}, W_{2}, W_{3}\right)}\right\|
\end{aligned}
$$

In which, $\theta_{\left(W_{1}, W_{2}, W_{3}\right)}$ denotes the finite area, which is enclosed by three points $\left(W_{1}, W_{2}, W_{3}\right)$, and it is a triangle area. Namely, $\theta_{\left(W_{1}, W_{2}, W_{3}\right)}$ can be represented as follow:

$$
\theta_{\left(W_{1}, W_{2}, W_{3}\right)}=\left\{Y \mid Y=\alpha_{2}\left[\alpha_{1}, W_{1}+\left(1-\alpha_{1}\right) W_{2}\right]+\left(1-\alpha_{2}\right) W_{3}, \alpha_{1} \in[0,1], \alpha_{2} \in[0,1]\right\} 4
$$

Then, $\Phi\left(X, W_{1}, W_{2}, W_{3}\right)-T h$ actually is the Euclid distance from $X$ to the triangle area of the $p S i 3$ neuron. The model of activation function is:

$$
f(x)=\left\{\begin{array}{l}
1, \quad x \leq T h \\
-1, \quad x>T h
\end{array}\right.
$$

In multi-dimensional space, we use every three sample's points of the same class to construct a finite 2D plane, namely, a triangle. Then several $2 \mathrm{D}$ spaces can be constructed, and we cover these planes by the pSi3 neuron to approximate the complicated "shape", which is formed by many sample points of the iris in multi-dimensional space.

\subsection{Construction of Complex Geometrical Shape}

Step 1: Let the sample points of the training set are $\alpha=\left\{A_{1}, A_{2}, \cdots, A_{N}\right\}$. In which, $\mathrm{N}$ is the number of the total sample points. To figure out the distance of every two points, the two points having the least distance are defined as $B_{11}$ and $B_{12}$. Let $B_{13}$ denotes the nearest point away from $B_{11}$ and $B_{12}$, and $B_{13}$ must doesn't in the line formed by $B_{11}$ and $B_{12}$. In this way, $B_{11} 、 B_{12}$ and $B_{13}$ construct the first triangle plane represented as $\theta_{1}$, which is covered by a $p S i 3$ neuron, the covering area is:

$$
P_{1}=\left\{X \mid \rho_{X \theta_{1}} \leq T h, X \in R^{n}\right\}
$$




$$
\theta_{1}=\left\{Y \mid Y=\alpha_{2}\left[\alpha_{1} B_{11}+\left(1-\alpha_{1}\right) B_{12}\right]+\left(1-\alpha_{2}\right) B_{13}, \alpha_{1 .} \in[0,1], \alpha_{2} \in[0,1]\right\} 7
$$

Where $\rho_{X \theta_{1}}$ denotes the distance from $X$ to $\theta_{1}$.

Step 2: Firstly, The rest points contained in $P_{1}$ should be removed. Then, according to the method of step1, define the nearest point away from $B_{11}$, $B_{12}$ and $B_{13}$ as $B_{21}$. Among $B_{11} 、 B_{12}$ and $B_{13}$, two nearest points away from $B_{21}$ are denoted as $B_{22}$ and $B_{23}$. And $B_{21} 、 B_{22}$ and $B_{23 i 3}$ construct the second triangle defined as ${ }_{2}$, which is covered by another ${ }^{p S i 3}$ neuron. And the covering area is described as follow:

$$
\begin{aligned}
& \qquad P_{2}=\left\{X \mid \rho_{X \theta_{2}} \leq T h, X \in R^{n}\right\} \\
& \theta_{2}=\left\{Y \mid Y=\alpha_{2}\left[\alpha_{1} B_{21}+\left(1-\alpha_{1}\right) B_{22}\right]+\left(1-\alpha_{2}\right) B_{23}, \alpha_{1} \in[0,1], \alpha_{2} \in[0,1]\right\} \\
& \text { Where } \rho_{X \theta_{2}} \text { denotes the distance from } X \text { to } \theta_{2} .
\end{aligned}
$$

Step 3: Remove the rest points contained in the covering area of the front $(i-1) p S i 3$ neurons. Let $B_{i 1}$ denotes the nearest point from the remained points to the three vertexes of the $(i-1)$ th triangle. Two nearest vertexes of the $(i-1)$ triangle away from $B_{i 1}$ are represented as $B_{i 2}$ and $B_{i 3}$. Then, $B_{i 1} 、 B_{i 2}$ and $B_{i 3}$ construct the $i t h$ triangle, defined as $\theta_{3}$.

In the same way, $\theta_{\hat{\beta}}$ is covered by a $p S i 3$ neuron. The covering area is

$$
\begin{array}{cc}
P_{i}=\left\{X \mid \rho_{X \theta_{2}} \leq T h, X \in R^{n}\right. & 10 \\
\theta_{3}=\left\{Y \mid Y=\alpha_{2}\left[\alpha_{1} B_{i 1}+\left(1-\alpha_{1}\right) B_{i 2}\right]+\left(1-\alpha_{2}\right) B_{i 3}, \alpha_{1,} \in[0,1], \alpha_{2} \in[0,1]\right\} & 11
\end{array}
$$

Step 4: Repeat the step 3 until all sample points are conducted successfully. Finally, there are $m p S i 3$ neurons, and their mergence about covering area is the covering area of every iris' class.

$$
P=\bigcup_{i=1} P_{i}
$$

\subsection{Iris recognition algorithm}

Taking $T h=0$ under recognition, the $p S i 3$ neuron can be described as follow:

$$
\rho=\left\|X-\theta_{\left(W_{1}, W_{2}, W_{3}\right)}\right\|
$$

The output $\rho$ is the distance from $X_{1}$ to the finite area $\theta_{\left(W_{1}, W_{2}, W_{3}\right)}$.

The distance from $X$ to the covering area of the $i t h$ class iris is:

$$
\rho_{i}=\min _{j=1}^{M_{i}} \rho_{\mathrm{ij}}, i=1, \cdots, 80
$$

In which, $M_{i}$ denotes the number of the pSi3 neuron of the ith iris, $\rho$ is the distance from $X$ to the covering area of the $j t h$ neuron of the $i t h$ class' iris.

The $X$ will be classified to the iris class corresponding to the least $\rho_{i}$. Namely, the classification method is: 


$$
j=\min _{i=1}^{80} \rho_{i}, \quad j \in(1, \cdots, 80)
$$

\subsection{Experimental results}

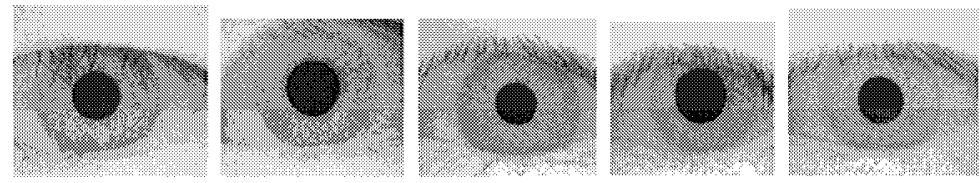

Fig. 3 iris samples from the training set
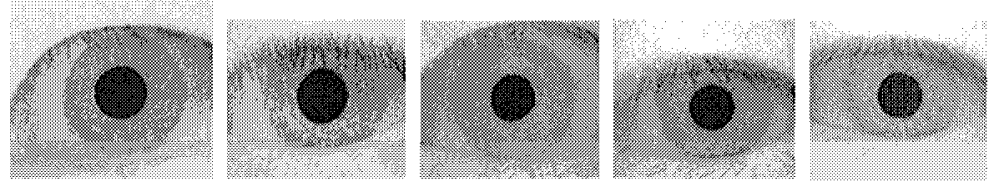

Fig. 4 iris samples from the second test set
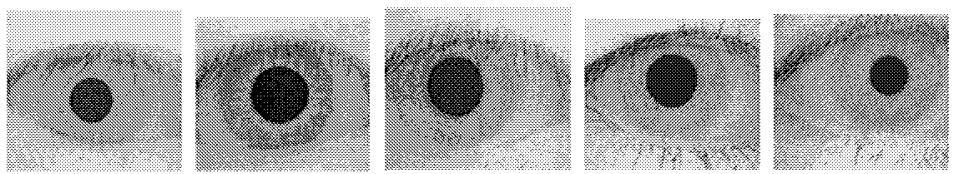

Fig. 5 iris samples from the first test set

Images of CASIA (Institute of Automation, Chinese Academy of Sciences) iris image database are used in this paper. The database includes 742 iris images from 106 different eyes (hence 106 different classes) of 80 subjects. For each iris class, images are captured in two different sessions and the interval between two sessions is one month. The experiment processes and experiment results are presented as follow:

(1) In our experiment, 3 random samples from each class in the frontal 80 classes (hence, 240 samples) are chosen for training, and a pSi3 neuron of multi weight neuron is constructed for the 3 samples. Five samples from the training set are shown in Fig.3. Then, the entire iris database is taken as test sample set. In which, $182(26 \times 7)$ samples, which don't belong to the classes of training samples, are referred to the first sample set. The remainder of total $560(80 \times 7)$ samples is referred to the second sample set. Fig. 4 shows five samples from the second test set and Fig. 5 shows five samples from the first test set.

(2) The correct rejection rate=the number of samples which are rejected correctly in the first sample set/the total number of the first sample set. The correct cognition rate=the number of samples which are recognized corrected in the second sample set / the total number of the second sample set. The error recognition rate $=$ (the number of samples which are 
recognized mistakenly in the first sample set +the number of samples which are recognized mistakenly in the second sample set) / the total number of the second sample set.

(3) For total 742 test samples, 180 samples are rejected correctly and the other 2 samples are recognized mistakenly in the first test sample; and 536 samples are recognized correctly and the rest 24 samples are recognized mistakenly in the second test sample. Therefore, the correct rejection rate is $98.9 \%(180 / 182)$, the correct cognition rate and the error recognition rate are $95.71 \%(536 / 560)$ and $3.5 \%((2+24) / 742)$ respectively.

\subsection{Experimental analysis}

We can conclude from the above experimental results that:

(1) Irises are trained as "cognition" one class by one class in our method, and it doesn't influence the original recognition knowledge for samples of the new added class.

(2) Although the correct cognition rate is not very well, the result of the correct rejection rate is wonderful. In our experiment, the correct rejection rate is $98.9 \%$, namely, the iris classes that don't belong to the training test can be rejected successfully.

(3) The iris recognition algorithm based on neuron of multi weight neuron is applied in the experiment and the total samples of every class construct the shape of 1D distribution. Namely, it is the network connection of different neuron.

(4) In above experiment, if the image preprocessing is more perfectly, the experimental results maybe better.

To sum up, it proves the proposed iris recognition algorithm based on multi-weighted neuron is effective.

\section{CONCLUSION}

Biomimetic Pattern Recognition using the analysis method of ANN, which is based on a novel all-purposed mathematic model of Multi weight neurons, is proposed in this Paper. It is a fire-new theoretics of Pattern Recognition. As the base, we have proposed a new model of Multi weight neuron and implemented it in the new CASSANDRA-II neurocomputer.

This method of pattern recognition is more effective and efficient than traditional pattern recognition. And it's application is very broad, besides "multi-camera human-face personal identification system" and "speech recognition", "Iris recognition" we also apply Biomimetic Pattern 
Recognition to identify objects (such as naval ship, tank, bus, bow, horse, sheep) from different directions on horizontal plane or sea level, etc.

From above analysis, the theory of Biomimetic Pattern Recognition using ANN and the method of multi weight neuron has created a new hopeful way for Pattern Recognition. This method is being improved and developed, and will be used practically into more science fields in future.

\section{National Natural Science Foundation of China: 60135010}

\section{REFERENCES:}

1. Wang Shou-jue, "Biomimetic (Topological) Pattern Recognition — A New Model of Pattern Recognition Theory and Its Applications" (in Chinese), ACTA ELECTRONICA SINICA, Vol.30, No.10, pp.1417-1420, Oct.2002

2. Wang Shou-jue, "Analysis and Theory of High-Dimension Space Geometry to Artificial Neural Networks", ACTA ELECTRONICA SINICA, Vol.30, No.1, pp.1-4, Jan.2002

3. WANG Shoujue, Biomimetics Pattern Recognition, Neural Networks Society (INNS, ENNS, JNNS) Newsletter, Vol.1 No.1, 3-5, March 2003

4. WANG Shoujue, WANG Bainan, Analysis and Theory of High-Dimension Space Geometry for Artificial Neural Networks (in Chinese), Acta Electronica Sinica, Vol.30 No.1, Jan 2002

5. WANG Shoujue, XU Jian, WANG Xianbao, QIN Hong, Multi-camera Human-face Personal Identification System Based on the BIOMIMETIC PATTERN RECOGNITION (in Chinese), Acta Electronica Sinica, Vol. 30 No. 1, Jan 2002

6. Wang Shoujue, Shi Jingpu, Chen Chuan, Li Yujian. Direction-Basis-Function Neural Networks [A].\#58 Session:4.2, IJCNN'99.[C] [6]

7. Wang Shou-jue, "Discussion on the Basic Mathematical Models of Neurons in General Purpose Neurocomputer", ACTA ELECTRONICA SINICA, Vol.29, No.5, pp.577-580, May. 2001

8. J.Daugman, Biometric Personal Identification System Based on Iris Analysis [P]. U.S. Patent 5291560, 1994.

9. J.Daugman, High Confidence Visual Recognition of Persons by a Text of Statistical Independence, IEEE Trans. Pattern Anal. Mach. Intell. 15(11) (1993) 1148-1161.

10.R.Wildes, Iris Recogniton: An Emerging Biometric Techonology, Proc. IEEE 85(1997) 1348-1363.

11. W.Boles, B. Boashash, A Human Identification Technique Using Image of the Iris and Wavelet Transform, IEEE Trans. Signal Process. 46(4) (1998) 1185-1188.

12. Wang Shoujue, Li Zhaozhou, Chen Xiangdong, Wang Bainan, Discussion on the Basic Mathematical Models of Neurons in General Purpose Neurocomputer, ACTA ELECTRONICA SINICA. 2001, 29(5): 577-580

13. Wenming Cao, Feng Hao, Shoujue Wang: The application of DBF neural networks for object recognition. Inf. Sci. 160(1-4): 153-160 (2004)

14. T. Risse, Hough Transform for Line Recognition, Computer Vision and Image Processing, $1989,46,327-345,1989$ 